\title{
Failure of matrix metalloproteinase-9 dimer induction by phorbol 12-myristate 13-acetate in normal human cell lines
}

\author{
MOHD WAHEED ROOMI, TATIANA KALINOVSKY, MATTHIAS RATH and ALEKSANDRA NIEDZWIECKI \\ Dr Rath Research Institute, Santa Clara, CA 95050, USA
}

Received June 5, 2014; Accepted February 19, 2015

DOI: $10.3892 / \mathrm{ol} .2015 .3132$

\begin{abstract}
Increasing experimental and clinical data has identified an association between increased levels of matrix metalloproteinase (MMP)-9 and shortened patient survival, cancer progression and metastasis. MMP-9 has a significant role in tumor cell invasion and metastasis, as it digests the basement membrane and components of the extracellular matrix. MMP-9 is secreted in either a monomeric or dimeric form. Although limited evidence exists concerning MMP-9 dimers, certain studies have demonstrated that the dimer is associated with aggressive tumor progression. This is believed to be due to the fact that cellular migration depends upon the MMP-9 dimer, and not the monomer. Our previous study revealed that cancer cell MMP-9 dimer secretion patterns could be divided into different categories, and that high MMP-9 and MMP-9 dimer secretion levels were correlated with the most aggressive cancer cell lines. It has been established that signal transduction pathways and cytokines, including those activated by phorbol 12-myristate 13-acetate (PMA), regulate the expression of MMPs. The aim of the present study was to analyze the expression patterns of MMP-2, MMP-9 and MMP-9 dimer in normal human cells from a number of tissues treated with PMA. Muscle, epithelial and connective tissues were selected for use in the present study, since adenosarcomas, carcinomas and sarcomas are derived from these tissue types, respectively. The cell lines were first cultured in 24-well tissue culture plates containing recommended media that was supplemented with $10 \%$ fetal bovine serum and antibiotics. When at confluency, the cells were washed and fresh medium was added. In addition, a parallel set of cultures was treated with PMA. Subsequent to a 24-h incubation period, the media were collected and analyzed using gelatinase zymography for the expression of MMP-2 and MMP-9 monomer and dimer forms. The results revealed that the cellular expression of MMP-2 and MMP-9 was dependent upon the primary tissue subtype. All cell lines, regardless of tissue origin, expressed MMP-2. PMA induced the expression of MMP-9 in muscle tissue, glandular
\end{abstract}

Correspondence to: Dr Aleksandra Niedzwiecki, Dr Rath Research Institute, 1,260 Memorex Drive, Santa Clara, CA 95050, USA

E-mail: author@drrath.com

Key words: matrix metalloproteinase-9 dimers, normal human cell lines, phorbol 12-myristate 13-acetate epithelia and supportive connective tissue cell lines. By contrast, cell lines of endothelial origin and proper connective tissue were insensitive to treatment with PMA. MMP-9 dimer secretion was not observed in any of the cell lines, which indicated that cellular migration is not supported by these cells.

\section{Introduction}

Increasing experimental and clinical data has identified an association between increased levels of matrix metalloproteinase (MMP)-9, a type IV collagenase, and shortened patient survival, cancer progression and metastasis. MMP-9 has a significant role in tumor cell invasion and metastasis, as it digests the basement membrane and components of the extracellular matrix (1-6). In addition to proteolysis, MMP-9 has been identified to have a significant role in cellular migration $(7,8)$. A unique characteristic of MMP-9 is its ability to be secreted in monomeric and disulfide-bonded dimeric forms. A study by Dufour et al (7) indicated that dimerization of MMP-9 through the hemopexin domain is required for MMP-9 enhanced cellular migration. Our previous study investigated the secretion patterns of the MMP-9 monomer and dimer in a number of cancer cell lines, and identified varying degrees of dimer secretion (8). High MMP-9 and MMP-9 dimer secretion levels were associated with the most aggressive cancer cell lines (8).

As tumor invasion is dependent upon cellular migration, and MMP-9 dimer secretion has been demonstrated to be required for cellular migration (7), the present study investigated MMP-9 monomer and dimer secretion patterns in a number of normal human cells from various tissues. It has been established that signal transduction pathways and cytokines, including those activated by phorbol 12-myristate 13-acetate (PMA), regulate the expression of MMPs. Therefore, the aim of the present study was to analyze the expression patterns of MMP-2, MMP-9 and MMP-9 dimer in normal human cells from a number of tissues treated with PMA. Muscle, epithelial and connective tissue tissues were selected for use in the present study, since adenosarcomas, carcinomas and sarcomas are derived from these tissue types, respectively.

\section{Materials and methods}

Cancer cell lines and reagents. In total, 14 normal human cell types obtained from three primary tissues, namely 
Table I. MMP-2, MMP-9 and MMP-9 dimer secretion patterns of normal human cells treated with $100 \mathrm{ng} / \mathrm{ml}$ phorbol 12-myristate 13-acetate.

\begin{tabular}{llccc}
\hline Cell line & \multicolumn{1}{c}{ Tissue } & MMP-2 & MMP-9 & MMP-9 dimer \\
\hline Dermal fibroblast (NHDF) & Stromal connective tissue & + & - & - \\
Lung fibroblast (NHLF) & Stromal connective tissue & + & - & - \\
Gingival fibroblast (HGF) & Stromal connective tissue & + & - & - \\
Synovial fibroblast & Stromal connective tissue & + & - & - \\
Prostate stromal (PrSC) & Stromal connective tissue & + & - & - \\
Vein endothelium (HUVEC) & Endothelial epithelial tissue & + & - & - \\
Aortic smooth muscle cells (Ao-SMC) & Smooth muscle tissue & + & - & - \\
Chondrocytes (NHAC-Kn) & Supportive connective tissue & + & + & - \\
Osteoblasts (NHOst) & Supportive connective tissue & + & + & - \\
Keratinocytes (NHEK-Ad) & Glandular epithelial tissue & + & + & - \\
Skeletal muscle (SK-SMC) & Striated muscle tissue & + & + & - \\
Hepatocytes & Glandular epithelial tissue & + & + & - \\
Bronchial smooth muscle & Smooth muscle tissue & + & + & - \\
Uterine smooth muscle & Smooth muscle tissue & + & + & -
\end{tabular}

MMP, matrix metalloproteinase.

epithelial, connective and muscle tissues, were analyzed in the present study. The cell lines, in addition to their recommended media, were purchased from Lonza (Walkersville, MD, USA), with the exception of the kidney parenchyma, gingival cells and hepatocytes, which were obtained from the American Type Culture Collection (Manassas, VA, USA) along with their recommended media, respectively. The human rheumatoid synovial fibroblasts were isolated from rheumatoid arthritic membranes following enzymatic dissociation using trypsin-EDTA (Gibco Life Technologies, Carlsbad, CA, USA). All other high-grade reagents, including fetal bovine serum (FBS), PMA, streptomycin and penicillin, were purchased from Sigma-Aldrich (St. Louis, MO, USA).

Cell culture. First, the cells were grown in their respective recommended media containing $10 \%$ FBS, $100 \mathrm{mg} / \mathrm{ml}$ streptomycin and $100 \mathrm{U} / \mathrm{ml}$ penicillin. Next, the cells were plated at a density of $1 \times 10^{5}$ cells $/ \mathrm{ml}$ in triplicate in 24-well tissue culture plates (Coster, Cambridge, MA, USA) and allowed to grow to confluency at $37^{\circ} \mathrm{C}$ and $5 \% \mathrm{CO}_{2}$ in a humidified atmosphere. The serum-supplemented medium was removed and the cell monolayer was washed twice with phosphate-buffered saline and then once with the recommended serum-free medium. Next, the cells were cultured for $24 \mathrm{~h}$ in an incubator with $0.5 \mathrm{ml}$ serum-free medium. The parallel sets of cultures were treated with $100 \mathrm{ng} / \mathrm{ml}$ PMA for the induction of MMP-9. The resulting conditioned media were then collected separately, pooled and centrifuged at $4^{\circ} \mathrm{C}$ at $704 \mathrm{xg}$ for $10 \mathrm{~min}$ in order to remove the cells and cellular debris. Finally, the supernatant was collected and used for gelatinase zymography, a highly-sensitive assay for gelatinolytic enzymatic activity, which is able to detect pro and active forms of MMP-2 and MMP-9.

Gelatinase zymography. Gelatinase zymography was performed under non-reducing conditions with $10 \%$ Novex
Pre-Cast SDS polyacrylamide gel (Invitrogen Life Technologies, Carlsbad, CA, USA) and $0.1 \%$ gelatin. In total, $20 \mu \mathrm{l}$ of each culture medium was mixed with sample buffer and loaded onto gels for SDS-PAGE with Tris-glycine-SDS buffer, according to the manufacturer's instructions (Thermo Fisher Scientific, Waltham, MA, USA). The samples were not boiled prior to electrophoresis. Subsequent to electrophoresis, the gels were washed twice in $2.5 \%$ Triton X-100 at room temperature for $30 \mathrm{~min}$ in order to remove the SDS. Next, the gels were incubated overnight at $37^{\circ} \mathrm{C}$ in a substrate buffer containing $50 \mathrm{mM}$ Tris- $\mathrm{HCl}$ and $10 \mathrm{mM} \mathrm{CaCl}_{2}$ at $\mathrm{pH} 8.0$, stained with $0.5 \%$ Coomassie Blue R250 in $10 \%$ glacial acetic acid and $50 \%$ methanol for $30 \mathrm{~min}$, and then destained. Subsequent to renaturation of the enzyme, the gelatinases digested the gelatin contained within the gel, which produced clear bands against an intensely-stained background. In addition, protein standards were run and approximate molecular weights were established by plotting the relative mobilities of known proteins.

\section{Results}

Normal human cell lines express MMP-2, but not MMP-9. The human dermal fibroblasts, gingival fibroblasts, lung fibroblasts, prostate stromal cells, smooth muscle cells, synovial fibroblasts and human vein endothelium cells secreted MMP-2 only, even when treated with $100 \mathrm{ng} / \mathrm{ml}$ PMA, as shown in Table I. Zymograms of MMP secretion by representative cell lines, human gingival fibroblasts and human prostrate stromal cells, are shown in Fig. 1.

Normal human cells secrete MMP-2 and MMP-9 upon stimulation by PMA, but fail to form MMP-9 dimers. The human chondrocytes, osteoblasts, skeletal muscle cells, hepatocytes, bronchial muscle cells, uterine muscle cells and keratinocytes secreted MMP-2 and MMP-9 following 
A

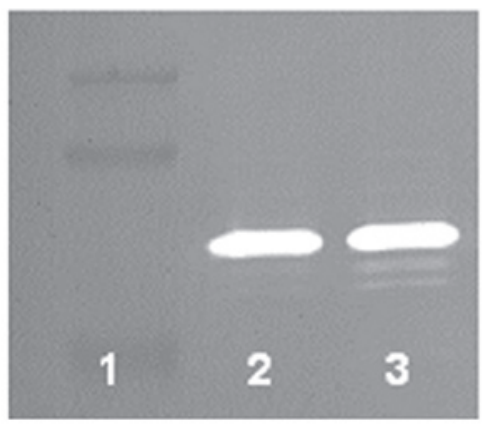

B

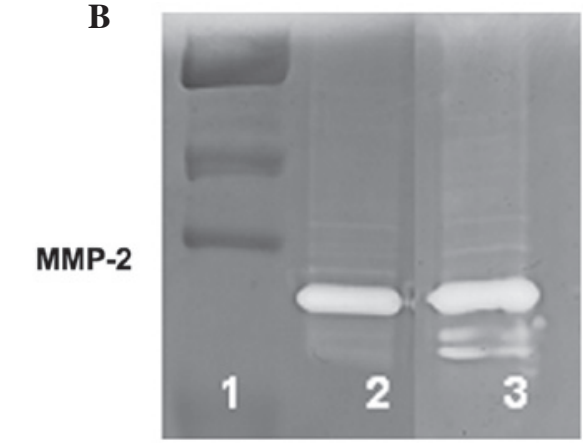

Figure 1. Gelatinase zymograms revealing normal human cells treated with 100 ng/ml PMA that secreted MMP-2, but not MMP-9. (A) Gingival cells (HGF) and (B) prostate cells (PrSC). Lanes: 1, markers; 2, control cells; 3, 100 ng/ml PMA-treated cells. Molecular weight MMP-2, 72 kDa. PMA, phorbol 12-myristate 13-acetate; MMP, matrix metalloproteinase.



Figure 2. Gelatinase zymograms revealing normal human osteoblasts treated with $100 \mathrm{ng} / \mathrm{ml}$ PMA that secreted MMP-2 and MMP-9. Lanes: 1, markers; 2, control cells; 3, 100 ng/ml PMA-treated cells. Molecular weight MMP-2, $72 \mathrm{kDa}$; molecular weight MMP-9, $94 \mathrm{kDa}$. PMA, phorbol 12-myristate 13-acetate; MMP, matrix metalloproteinase.

treatment with PMA, but failed to secrete MMP-9 dimers, as shown in Table I. Zymograms of MMP secretion by human osteoblasts, are shown in Fig. 2.

\section{Discussion}

Normal human stromal connective tissue cells (dermal fibroblasts, lung fibroblasts, gingival fibroblasts, synovial fibroblasts and prostate stromal cells), endothelial epithelial tissue cells (vein endothelium cells) and aortic smooth cells treated with PMA were found to secrete MMP-2, but not MMP-9 monomer or dimer in the present study. By contrast, normal human supportive connective tissue cells (chondrocytes and osteoblasts), striated muscle cells from skeletal muscle), epithelial glandular tissue cells (hepatocytes and keratinocytes) and smooth muscle cells from bronchial and uterine tissues were found to secrete MMP-2 and MMP-9 following treatment with PMA, but failed to secrete MMP-9 dimer.

Our previous study investigated the relative secretion patterns of MMP-9 monomer and dimer by a variety of carcinomas, sarcomas, adenosarcomas and leukemia cell lines with and without PMA induction (8). It was identified that cancer cells derived from the same connective tissues as those analyzed in the present study, namely fibrous tissue (fibrosarcoma HT-1080), cartilage tissue (chondrosarcoma SW-1353) and bone tissue (osteosarcoma U-2OS), exhibited MMP-9 dimer secretion subsequent to treatment with PMA.
Among the tissue cancer cell lines, sarcomas exhibited the highest levels of MMP-9 monomer and dimer secretion. In particular, osteosarcoma cells demonstrated the highest MMP-9 to MMP-9 dimer ratio, which indicated an extremely aggressive form of cancer. Muscle tissue-derived sarcomas, including leiomyosarcoma SK-UT-1 (smooth muscle) and rhabdomyosarcoma (striated muscle) also secreted MMP-9 dimers, but with a slightly lower level of MMP-9. Similarly, epithelial tissue cancers, including adenocarcinomas (hepatocellular carcinoma SK-Hep-1 and renal cell carcinoma RCC 786-0) secreted MMP-9 dimers, but at a lower level of MMP-9.

The aim of the present study was to analyze the expression patterns of MMP-2, MMP-9 and MMP-9 dimer in normal human cells from a number of tissues treated with PMA. As expected, none of the normal human cells that were analyzed secreted MMP-9 dimer, which indicated that cellular migration was not supported by these cells. Therefore, it can be concluded that the MMP-9 dimer appears to be associated with cancer cells.

\section{Acknowledgements}

The present study was funded by a grant from the Dr Rath Health Foundation (Santa Clara, CA, USA), which is a non-profit organization.

\section{References}

1. Kleiner DE and Stetler-Stevenson WG: Matrix metalloproteinases and metastasis. Cancer Chemother Pharmacol 43 (Suppl): S42-S51, 1999.

2. Yurchenko PD and Schitny JC: Molecular architecture of basement membranes. FASEB J 4: 1577-1590, 1990.

3. Barsky SH, Siegel GP, Jannotta F and Liotta LA: Loss of basement membrane components by invasive tumors but not by their benign counterparts. Lab Invest 49: 140-147, 1983.

4. Liotta LA, Tryggvason K, Garbisa S, et al: Metastatic potential correlates with enzymatic degradation of basement membrane collagen. Nature 284: 67-68, 1980.

5. Stetler-Stevenson WG: The role of matrix metalloproteinases in tumor invasion, metastasis and angiogenesis. Surg Oncol Clin N Am 10: 383-392, 2001.

6. Stetler-Stevenson WG: Type IV collagenases in tumor invasion and metastasis. Cancer Metastasis Rev 9: 289-303, 1990.

7. Dufour A, Zucker S, Sampson NS, et al: Role of matrix metalloproteinase-9 dimers in cell migration: Design of inhibitory peptides. J Biol Chem 285: 35944-35956, 2010.

8. Roomi MW, Kalinovsky T, Rath M and Niedzwiecki A: Effect of a nutrient mixture on matrix metalloproteinase-9 dimers in various human cancer cell lines. Int J Oncol 44: 986-992, 2014. 